\title{
Alimentation séparée (céréales graines entières + aliment complémentaire granulé + coquilles d'huître) des poulettes et des poules pondeuses en climat chaud
}

\author{
T. Yo ${ }^{1}$, M. Picard ${ }^{2}$, H. Guerin ${ }^{3}$, P. Dauvilliers ${ }^{4}$
}

YO (T.), PICARD (M.), GUERIN (H.), DAUVILLIERS (P.). Alimentation séparée (céréales graines entières + aliment complémentaire granulé + coquilles d'huitre) des poulettes et des poules pondeuses en climat chaud. Revue Élev. Méd. vét. Pays trop., 1995, 48 (1) : $67-76$

Trois cents poulettes Isabrown ont reçu, de 5 à 42 semaines, soit une alimentation complète $(\mathrm{AC})$ "témoin" en farine, soit une alimentation "séparée" (AS) en libre choix composée de maïs "graine entière", de coquilles d'huître et d'un aliment complémentaire granulé offert $a d$ libitum (ASL) ou rationné pendant la ponte (ASR : $30 \mathrm{~g} /$ poule/j). L'ingestion globale d'aliment et la croissance des poulettes n'ont pas été modifiées par le régime jusqu'au débecquage pratiqué à l'âge de 10 semaines. Celui-ci a réduit la proportion d'aliment complémentaire consommé par les poulettes en ASL de 53 à 17 p. 100 de l'ingéré total. Les poulettes en ASL avaient un poids vif moyen à 18 semaines inférieur à celui des poulettes $\mathrm{AC}$ (1524 et $1600 \mathrm{~g}$ respectivement) et sont entrées en ponte un peu plus tard (intensité de ponte à 21 semaines : 5 et 25 p. 100 respectivement). Pendant la période 23-42 semaines, les performances de ponte et les caractéristiques des reufs n'ont pas été significativement influencées par le régime alimentaire. Les quantités globales d'aliments consommés par les poules en AS étaient significativement inférieures à celles des poules témoins $\mathrm{AC}$. Par conséquent, les rendements énergétiques et protéiques des régimes étaient améliorés par l'alimentation séparée. Les poules ASL tendaient à surconsommer l'aliment compémentaire $(50 \mathrm{~g} / \mathrm{j} /$ poule au lieu de $30 \mathrm{~g} / \mathrm{j} /$ poule pour les poules ASR) sans amélioration des productions. Dans ces conditions, l'alimentation séparée rationnée permet une utilisation directe efficace des céréales produites localement en réduisant les frais de transport, de broyage, de mélange et de granulation de l'aliment des poules pondeuses.

Mots clés : Poule pondeuse - Technique d'alimentation - Ressource alimentaire - Croissance - Ponte - Caractéristique de l'cuf - Climat tropical - Côte d'Ivoire.

\section{INTRODUCTION}

Plusieurs études récentes soulignent les limites de l'utilisation d'un aliment complet $(\mathrm{AC})$ classique pour nourrir une poule chez qui la formation de l'œuf est un phénomène cyclique (34). L'existence chez les poules en ponte d'un appétit calcique spécifique a été largement démontrée $(25,26,35)$ et a conduit à l'utilisation de la méthode de complémentation calcique (CC). Cette méthode consiste à offrir aux poules un aliment pauvre en calcium, complémenté avec du calcium particulaire, généralement

1. IDESSA-DRA, BP 633 Bouaké, Côte d'lvoire.

2. INRA-SRA, 37380 Nouzilly, France.

3. CIRAD-EMVT, 10, rue Pierre Curie, 94704 Maisons-Alfort Cedex, France.

4. UFAC, 95450 Vigny, France.

Reçu le 9.11.1994, accepté le 7.3.1995. sous forme de coquilles d'huitre. Chez la poule en ponte, la CC entraîne une amélioration de la qualité de la coquille (épaisseur, index) par une ingestion calcique plus élevée et/ou l'augmentation de la proportion de calcium déposé au niveau de la coquille $(29,30)$. De plus, plusieurs études ont montré que la $\mathrm{CC}$ induit une augmentation de l'ingéré énergétique de la poule se traduisant, en climat chaud, par une amélioration du taux de ponte par rapport à l'aliment complet (31).

Si l'existence de l'appétit calcique de la poule pondeuse est bien établie, l'aptitude des volailles de souche ponte à s'adapter à une alimentation séparée (AS) offrant au choix une source énergétique, une source protéique et une source minérale ne semble pas encore clairement démontrée. Quelques auteurs ont montré que l'AS pendant la période pré-ponte n'a pas d'effets néfastes sur la croissance $(9,22,32)$ ni sur l'âge de maturité sexuelle des poulettes $(7,36,39)$. Cowan et Michie (8) et Karunajeewa et Tham (21) ont montré que l'utilisation de l'AS en période pré-ponte n'affecte pas non plus la ponte ultérieure de la poule. En revanche, l'AS semble induire chez la poulette une surconsommation des protéines, notamment à l'approche de la maturité sexuelle $(22,37)$.

Chez la poule en ponte, l'AS n'a pas d'incidence négative sur le taux de ponte $(14,20)$, le poids moyen de l'œuf (24) ou les proportions du jaune et du blanc de l'œuf (6). Toutefois, les auteurs signalent généralement une aptitude limitée de la poule, en situation de choix protéine/énergie, à réguler son ingéré alimentaire de manière adéquate sur une longue période. Plusieurs auteurs rapportent une surconsommation de protéines, sans effets positifs apparents sur la production d'œufs $(12,13,20,22)$, bien que Classen et Scott (7) signalent que les poules s'avèrent capables de réguler leur ingéré nutritionnel lorsque l'AS est introduite avant l'âge de maturité sexuelle.

Cette étude a été entreprise pour évaluer, en milieu tropical réel, l'effet de l'AS (avec du maïs non concassé) sur la croissance des poulettes, les performances de ponte, la qualité des œufs et le comportement alimentaire des poules. L'existence éventuelle d'une interaction entre les stratégies nutritionnelles (AS ou $A C$ ) utilisées successivement pendant les phases de pré-ponte et ponte est mesurée pour définir les conditions optimales d'adaptation des animaux à leur régime. 
TABLEAU I

Caractéristiques nutritionnelles des aliments utilisés au cours de l'essai
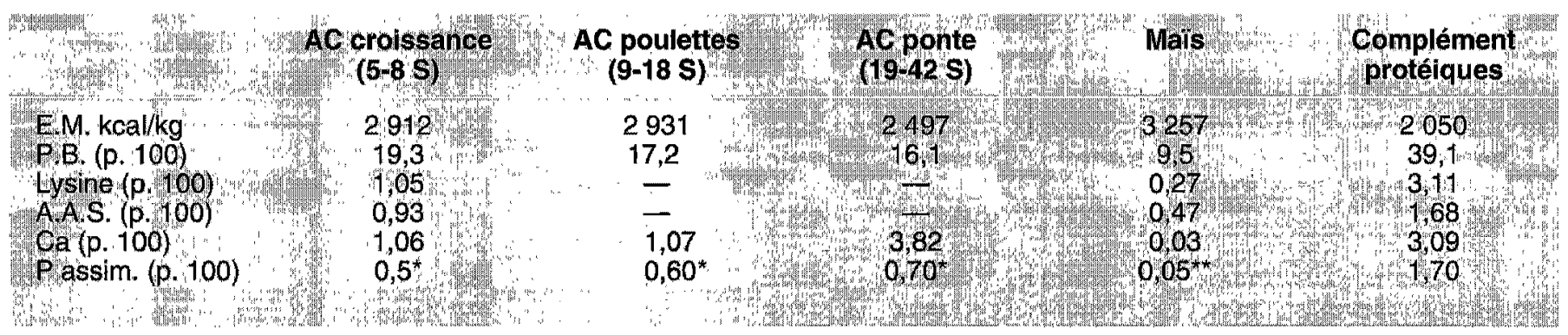

* Données communiquées par le fabricant (SIPRA).

** Données extraites des tables INRA (1984).

\section{MATÉRIEL ET MÉTHODES}

Une expérience a été conduite en 2 phases au Département des Ressources animales de l'IDESSA (Bouaké) du 9 juillet 1992 au 28 avril 1993 : la première phase de 0 à 18 semaines d'âge sur la croissance des poulettes, et une seconde phase de 19 à 42 semaines sur les performances de ponte. La première phase a eu lieu en saison des pluies avec des températures minimales (hebdomadaires) comprises entre 21,5 et $25,5^{\circ} \mathrm{C}$ et les maximales comprises entre 28 et $33^{\circ} \mathrm{C}$. La ponte a débuté en fin de saison des pluies et s'est poursuivie en saison sèche ; les températures minimales étaient alors de $20-24^{\circ} \mathrm{C}$ et les maximales de $30-35^{\circ} \mathrm{C}$ (fig. 1).

\section{Phase d'élevage des poulettes (0-18 S)}

Trois cents poussins de souche "Isabrown" ont été répartis en 10 groupes de 30 animaux dans des parquets de $6 \mathrm{~m}^{2}$ d'un poulailler semi-ouvert à ventilation statique. Tous les animaux ont reçu pendant les 4 premières semaines un aliment complet contenant 22,7 p.100 de protéines brutes et $2930 \mathrm{kcal}$ d'EM/kg. Pendant la période expérimentale 5-18 S, 2 modes d'alimentation à volonté ont été comparés : l'AS (maïs graines entières + complément protéique) et l'aliment complet $(A C)$ équilibré. Les caractéristiques nutritionnelles des aliments utilisés sont indiquées dans le tableau I. Les teneurs en EM ont été calculées avec l'équation de Janssen (19), pour le maïs, et l'équation CEE indiquée par Carré et Rozo (5) pour le complément protéique et les aliments complets.

Chaque régime ( $A S$ ou $A C$ ) a été distribué à 5 parquets de poulettes, soit 5 répétitions par traitement. Dans les parquets recevant le régime séparé (AS), le maïs et l'aliment complémentaire ont été distribués, séparément, à volonté dans 2 mangeoires siphoïdes disposées côte à côte au centre du parquet. Les consommations d'aliments ont été mesurées chaque semaine. Les poulettes ont été pesées individuellement à $2,4,6,8,10,12$ et 18 semaines d'âge. L'éclairement, continu pendant les 4 premiers jours, a été réduit à $18 \mathrm{~h}$ par nycthémère pour le reste de la période d'élevage. Au début de la dixième

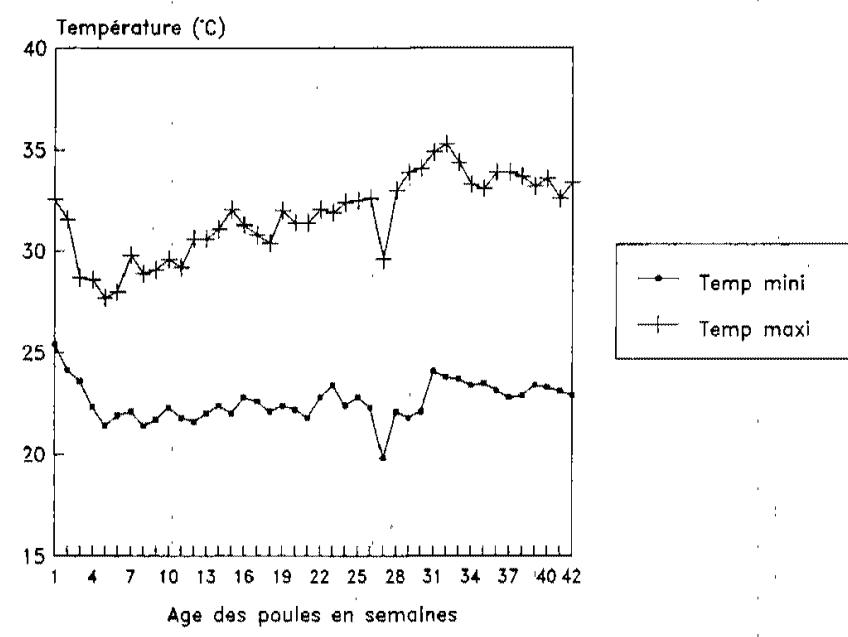

Figure 1: Evolution de la température au cours de l'expérience.

semaine d'âge, toutes les poulettes ont subi un débecquage. A la douzième semaine, les animaux ont été répartis en 18 lots de $16 / 17$ poulettes : 9 lots AC et 9 lots AS. Le type d'aliment reçu ( $A C$ ou $A S$ ) est resté le même pour chaque animal pendant toute la période expérimentale (5-18 S).

\section{Phase de ponte (19-42 S)}

La "phase ponte" a débuté avec la 19e semaine. Un dispositif à 2 facteurs croisés a été adopté : le facteur 1 correspond au régime alimentaire en phase d'élevage (5$18 \mathrm{~S}$ ) avec 2 niveaux (ac et as) et le facteur 2 au régime en phase de ponte avec 3 niveaux : aliment complet en farine $(A C F)$, aliment séparé avec complément protéique à volonté (ASL) et aliment séparé avec complément protéique rationné à $30 \mathrm{~g} / \mathrm{anim} . / \mathrm{j}$ (ASR). Ce dispositif devait permettre de mettre en évidence une interaction éventuelle entre le mode d'alimentation en phase d'élevage et l'alimentation en phase de ponte. II s'agit donc de comparer 6 stratégies alimentaires différentes: ACF (ac), ACF (as), ASL (ac), ASL (as), ASR (ac), ASR (as), les ali. ments reçus en période de croissance étant signalés entre parenthèses. 
Chaque traitement a été appliqué à 3 parquets de 16/17 poules. Dans chaque parquet d'AS, les animaux disposaient au choix de 3 aliments : le maïs graines entières, le complément protéique granulé, disponibles séparément dans 2 mangeoires siphoïdes et les coquilles d'huître en particules grossières de 4-6 mm distribuées dans une mangeoire linéaire de $60 \mathrm{~cm}$ de long.

Les poules ont été pesées à $18 \mathrm{~S}$ (début d'essai), $28 \mathrm{~S}$, $42 \mathrm{~S}$ d'âge (fin d'essai). Les consommations d'aliments ont été mesurées chaque semaine pour chaque parquet. La consommation d'eau a été mesurée toutes les 8 semaines pendant 2 jours consécutifs. Le nombre d'œufs pondus par parquet a été relevé quotidiennement et un taux de ponte moyen a été calculé pour chaque semaine et chaque période de 28 jours. A la fin de chaque période, pendant 3 jours consécutifs, tous les œufs ont été pesés individuellement. A $24 \mathrm{~S}, 28 \mathrm{~S}$ et $42 \mathrm{~S}$ d'âge, tous les œufs pondus au cours d'un nycthémère ont subi différentes mesures pour l'évaluation de la qualité : poids de l'œuf entier, poids du jaune, poids de la coquille et couleur du jaune. Le poids du jaune a été obtenu par pesée directe après séparation du blanc. La coquille entière a été lavée puis mise à l'étuve à $90^{\circ} \mathrm{C}$ pendant 12 $\mathrm{h}$ au moins avant d'être pesée. Le poids du blanc a été déduit par différence : poids du blanc = poids de l'œuf entier (poids du jaune + poids de la coquille). La couleur du jaune a été évaluée visuellement par comparaison avec l'échelle de "Roche" graduée de 1 à 15. Les paramètres relatifs à la quantité de coquille déposée ont été calculés suivant les équations indiquées par Bonnet et Mongin (3) ainsi que Sauveur (33) où :

- $S$ (surface de l'œuf) $=K^{\star} P^{2 / 3}$

avec $P=$ poids de l'œuf entier ; $K=$ coefficient $(4,67$ à 4,69)

- I (index de coquille) $=\mathrm{C} / \mathrm{S} * 100$

avec $\mathrm{C}=$ poids de la coquille

- e (épaisseur de la coquille) = 1/23,5.

\section{Analyse statistique}

Les données recueillies ont été analysées à l'aide du logiciel STAT-ITCF. Les données relatives à la phase de croissance (5-18 S) ont été soumises à une analyse de variance à un facteur (facteur aliment) à deux niveaux (ac et as). En revanche, les données de la phase de ponte ont subi une analyse multifactorielle : type d'aliment en croissance avec 2 niveaux (ac et as), type d'aliment pendant la ponte avec 3 niveaux (acf, asl et asr) ainsi que le stade de ponte/âge de la poule avec 6 niveaux. L'analyse a été faite "pas à pas" pour chacune des 6 périodes de ponte afin de vérifier la signification des interactions régime croissance * régime ponte. Les moyennes ont été comparées à l'aide du test de comparaison multiple de Newman et Keuls.

\section{RÉSULTATS}

\section{Phase pré-ponte}

\section{Gain de poids}

L'évolution du poids vif est représentée par la figure 2. Jusqu'à 10 semaines d'âge, le poids vif des poulettes est identique pour les 2 régimes alimentaires $(p>0,05)$. Cependant, au cours de la période 10-12 S, suite au débecquage, la croissance des animaux recevant l'AS a été plus lente ; ce qui a conduit à un poids vif à $12 \mathrm{~S}$ et 18 S significativement plus faible qu'avec l'AC ( $p<0,001$ ). A 18 semaines d'âge, le poids vif moyen est de $1600 \pm 155 \mathrm{~g}$ pour l'AC et $1524 \pm 159 \mathrm{~g}$ pour l'AS.

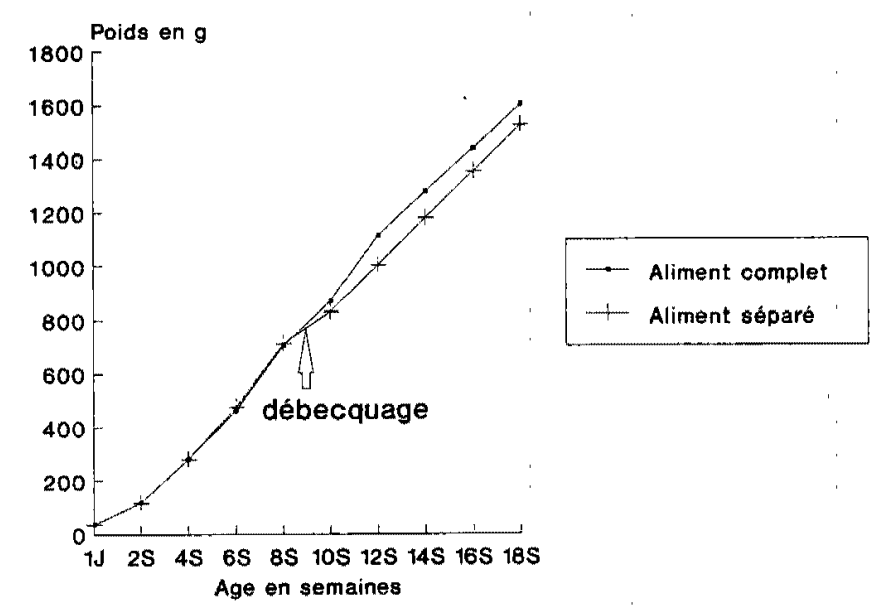

Figure $2:$ Evolution du poids vif des poulettes $(n=150 /$ traitement).

\section{Ingestion d'aliments et efficacité nutritionnelle}

Les ingestions d'aliments, d'énergie, de protéines et l'indice de consommation sont présentés dans le tableau Il. L'ingestion totale d'aliment évolue de manière comparable pour les 2 régimes jusqu'à la 9e semaine : de 16 g/anim./j au cours de la première semaine, elle atteint $99 \mathrm{~g}$ à la $9 \mathrm{e}$ semaine pour l'AC et $88 \mathrm{~g}$ pour l'AS. A la 10 e semaine, suite au débecquage, on observe une baisse brusque de la consommation. Chez les animaux recevant l'AS, cette baisse est due à une forte réduction de la consommation du complément protéique granulé. Les animaux retrouvent ensuite progressivement leur niveau d'ingestion antérieur ; cependant, cette reprise est plus lente pour les animaux en AS que pour les poulettes témoins recevant un aliment complet en farine (fig. 3 ).

En AS, la proportion de complément dans la ration quotidienne a été très variable. Au cours de la période de 5 à $9 \mathrm{~S}$, elle varie de 50 à 60 p.100 de la ration, sauf pendant l'adaptation à la 5 e semaine où il représente 40 p.100 de l'ingéré total. Ces taux correspondent à une teneur en 
TableaU II

Ingestion alimentaire et indices de consommation des poulettes $5-18 \mathrm{~S}(\mathrm{n}=150 /$ traitement $)$

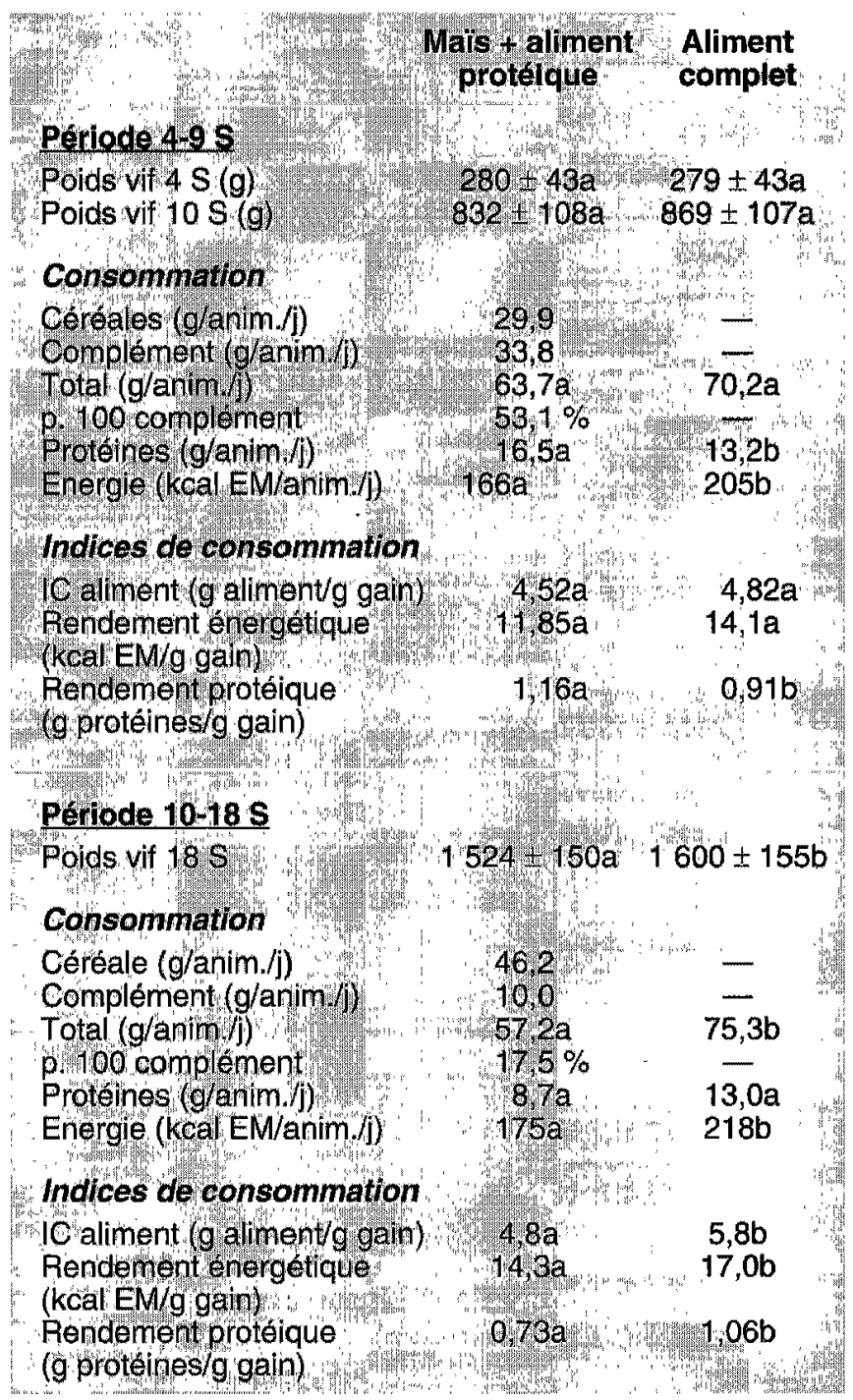

Les moyennes figurant sur la même ligne et portant la même lettre ne sont pas significativement différentes $(p>0,05)$.

protéines de 22 à 28 p.100 dans la ration ingérée. Après le débecquage, à $9 \mathrm{~S}$ d'âge, on observe un changement du comportement alimentaire des poulettes : l'ingestion du maïs continue d'augmenter tandis que la consommation de complément est réduite à environ 20 p.100 de l'ingéré total pour la période 10-18 semaines correspondant à une teneur en protéines de 15 à 16 p.100 dans la ration (fig. 4).

Les indices de consommation et l'efficacité protéique ne diffèrent pas significativement jusqu'à 9 semaines $(p>0,05)$; au-delà, ces indices, de même que l'efficacité protéique, sont meilleurs en AS qu'en $A C(p<0,0001)$.

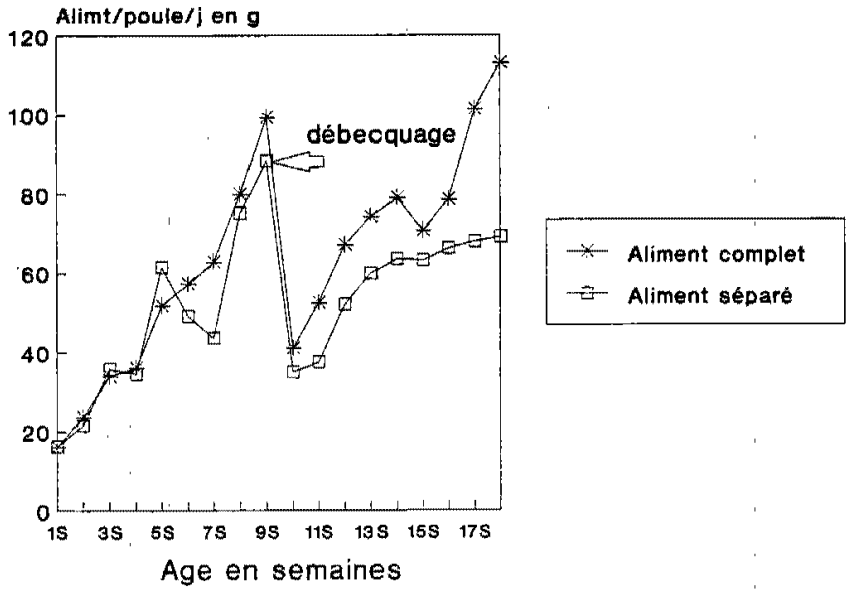

Figure 3 : Evolution de la consommation totale d'aliment de 0 à 18 semaines.

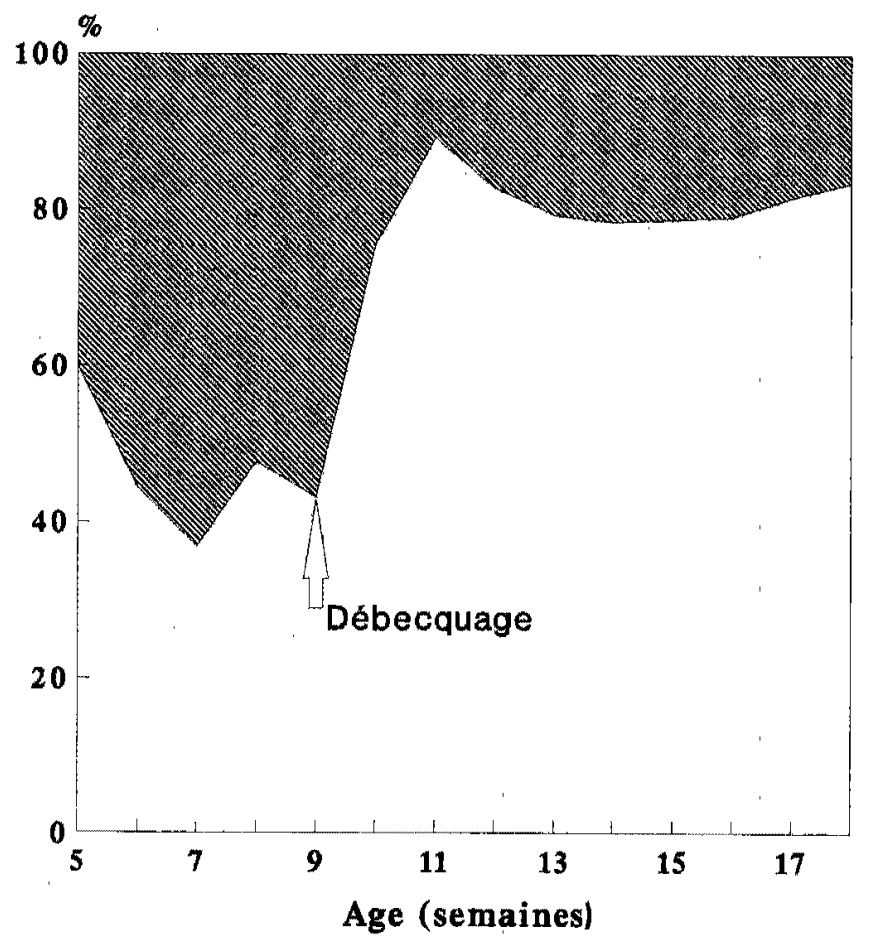

\section{Maïs}

\section{Complémentaire}

Figure 4 : Effet du debecquage sur le choix alimentaire des poulettes.

\section{Phase ponte}

\section{Production d'œufs}

L'évolution des taux de ponte est représentée par la figure 5. Les données relatives au poids moyen de l'œuf et à la masse d'œufs produite sont indiquées dans le tableau III.

Chez les animaux ayant reçu l'AS en période pré-ponte, on observe un retard de la date d'entrée en ponte : à $21 \mathrm{~S}$ 
Tableav III

Production d'œufs et évolution de quelques paramètres de qualité de l'œuf (19-42 S)

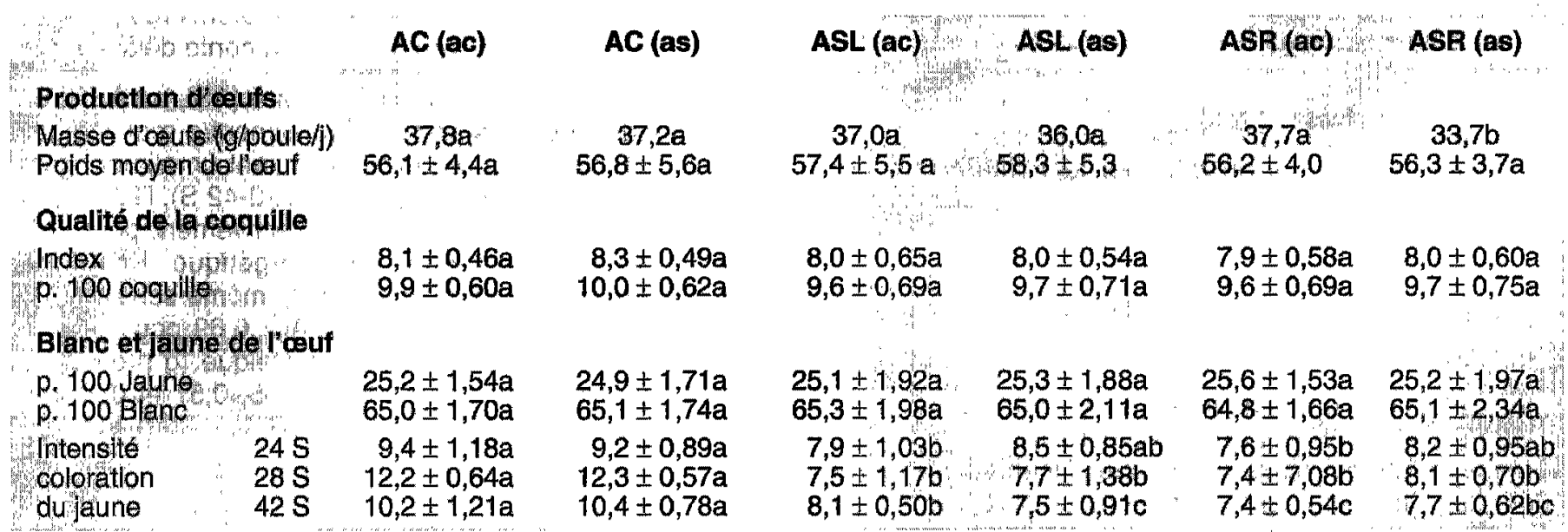

Les moyennes figurant sur la même ligne et portant la même lettre ne sont pas significativement différentes $(p>0,05)$.

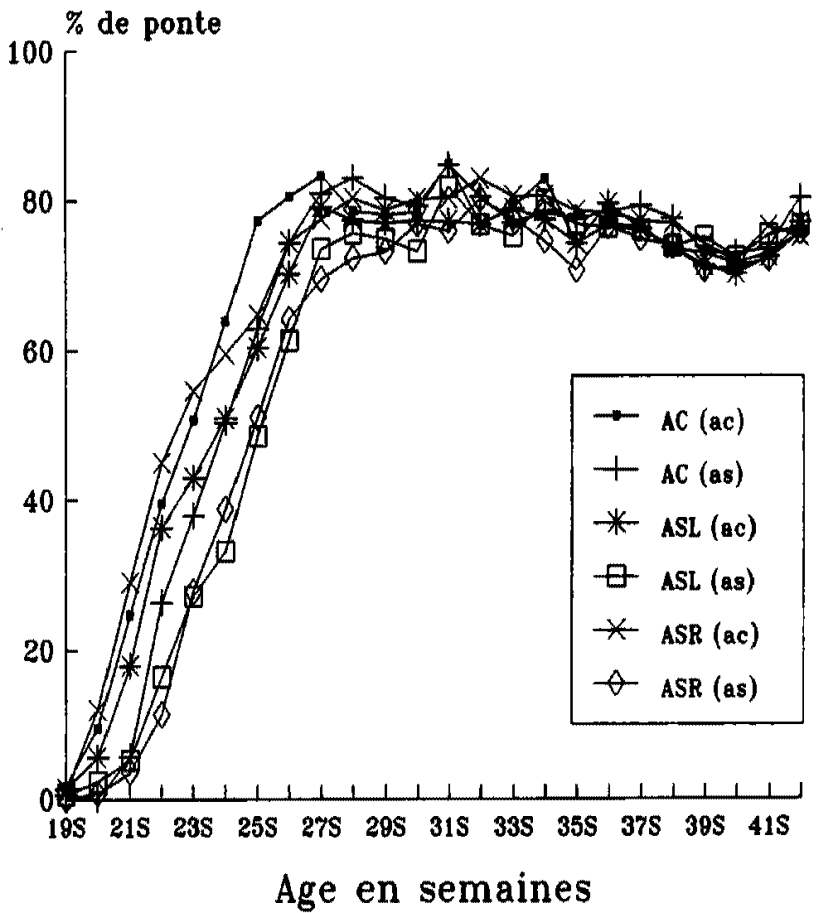

Figure 5 : Evolution du taux de ponte.

d'âge, ces poules ont un taux de ponte de 5 p.100 contre 25 p.100 pour celles ayant été nourries à l'AC. Compte tenu de ce décalage, on observe, pour les 2 premières périodes (19-26 S), une production d'œufs plus faible chez les poules nourries avec l'AS avant le début de la ponte $(p<0,001)$. Cet effet s'estompe par la suite. Pendant la période de ponte (27-42 S), le type d'aliment (AC, ASL ou ASR) n'a pas d'effet sur le taux de ponte ou la masse d'œufs produite $(p>0,05)$. En revanche, il a une influence significative sur le poids moyen de l'œuf $(p<0,001)$ : l'ASL donne des œufs plus lourds que l'AC et l'ASR. Cette différence apparaît à partir de la période 3 et persiste par la suite. L'alimentation avant la ponte n'affecte pas les poids moyens de l'œuf ( $p>0,05)$. L'analyse unifactorielle sur les 6 combinaisons alimentaires utilisées montre, pour l'ensemble de la période 19-42 $S$ que les masses d'œufs produites avec l'AC (ac), l'AC (as), l'ASL (ac), l'ASL (as) et l'ASR (ac) ne diffèrent pas significativement $(p>0,05)$. Seule la combinaison ASR (as) donne une production plus faible $(p<0,05)$. II n'y a pas d'interaction aliments pré-ponte/ponte sur la production d'œufs $(p>0,05)$.

\section{Paramètres de qualité de l'œuf}

Le tableau III indique l'influence des différentes combinaisons alimentaires sur quelques caractéristiques de l'œuf. D'une manière générale, on observe que le type d'alimentation n'affecte pas le pourcentage de jaune ou de blanc de l'œuf $(p>0,05)$. En revanche, le ratio jaune/poids total de l'œuf augmente avec l'âge de la poule $(p<0,001)$ tandis que la part du blanc diminue.

Le type de combinaison alimentaire utilisé n'a généralement pas eu d'effet significatif sur l'index ou le pourcentage de coquille ; toutefois, à $28 \mathrm{~S}$ d'âge, les œufs du traitement ASR (ac) ont présenté un index de coquille inférieur à ceux du traitement $\mathrm{AC}$ (as). Le pourcentage de coquille tend à diminuer avec l'âge passant de 9,99 à 9,72 et 9,49 p. 100 à 24,28 et 42 semaines respectivement.

Les poules consommant l'AC produisent des jaunes plus colorés $(p<0,001)$ que celles recevant l'ASL ou l'ASR. L'interaction aliment * âge observée sur la coloration du jaune est due à l'AC dont la composition en matières premières a dû varier au cours de l'expérimentation. 


\section{Evolution du poids vif des poules}

Les poules nourries pendant la ponte en régime ASL ont eu les gains de poids les plus élevés $(3,64 \mathrm{~g} / \mathrm{j})$ contre $2,78 \mathrm{~g} / \mathrm{j}$ pour l'ACF et $2,48 \mathrm{~g} / \mathrm{j}$ pour l'ASR. Les poules ayant reçu l'AS en phase pré-ponte ont eu, pendant la ponte, une croissance plus forte $(3,26 \mathrm{~g} / \mathrm{j}$ pour l'AS contre 2,67 pour l'AC) et ont pu rattraper leur retard pondéral après 10 semaines de ponte.

\section{Ingestion d'aliments et efficacité nutritionnelle}

L'ingestion moyenne d'aliments, de nutriments et les indices de consommation sont rapportés dans le tableau IV. Les poules recevant une alimentation séparée ingèrent, à tous les âges, nettement moins d'aliment que celles en $A C(p<0,001)$. Les poules recevant le complément protéique à volonté (ASL) consomment plus d'aliment que celles rationnées à $30 \mathrm{~g} / \mathrm{j}$ de complément. Le surplus de consommation est dû à une ingestion plus élevée de complément en régime libre. Chez les poules rationnées, l'ingestion de complément n'a effectivement atteint $30 \mathrm{~g} / \mathrm{j}$ qu'après 8 semaines ; par la suite, cette quantité de complément était consommée en totalité en 4 à $5 \mathrm{~h}$ après distribution.

Les quantités de maïs consommées pendant les 4 premières périodes de ponte en ASL et ASR sont identiques $(p>0,05)$. Ce qui indique que l'appétit pour le complément n'est pas reporté sur le maïs. Toutefois, une compensation partielle est apparue au cours des 2 dernières périodes où les animaux en ASR consomment 6 à $9 \mathrm{~g}$ de maïs de plus qu'en ASL. Les animaux habitués à l'AS en période pré-ponte consomment pendant la ponte un peu plus de mails que ceux ayant été élevés avec l'AC (3$6 \mathrm{~g} / \mathrm{anim} . \mathrm{j}$ ) ; cette différence s'estompe toutefois après les 4 premières périodes. La consommation du complément qui a représenté, en moyenne, 28 p.100 de la ration en ASR a été nettement plus élevée avec le régime ASL, notamment à partir de la période $26-30 \mathrm{~S}$ : 40 à $46 \mathrm{p} .100$.

Le régime alimentaire n'a pas affecté significativement la quantité d'eau bue par les poules $(p>0,05)$, probablement du fait de la forte variabilité inter-parquets. Toutefois, le ratio eau/aliment pour les régimes séparés $(2,6$ à 4) est deux fois supérieur à celui observé sur $A C(1,2$ à 1,8) $(\mathrm{p}<0,001)$ (tabl. V).

Pendant la période 23-42 S, l'aliment complet à volonté a entraîné une ingestion d'énergie élevée (386 kcal), supérieure à l'ASL (298 kcal) et à l'ASR (269 kcal). En revanche, le type de régime pré-ponte n'influence pas significativement ce paramètre $(p>0,05)$. Compte tenu du rationnement du complément protéique, les poules du traitement ASR ont eu la plus faible ingestion de protéines $(p<0,001): 17,9 \mathrm{~g} /$ poule/j contre $25,6 \mathrm{~g}$ pour l'ASL et $25,0 \mathrm{~g}$ pour l'AC. Dans l'ensemble, les animaux en $A C$ ont ingéré quotidiennement plus de calcium
$(5,90 \mathrm{~g})$ que ceux en ASL $(4,66 \mathrm{~g})$ ou en $\operatorname{ASR}(3,75 \mathrm{~g})$. L'effet de l'alimentation pré-ponte observé sur l'ingéré protéique et calcique au cours des 2 premières périodes (19-26 S) est dû au retard d'entrée en ponte déjà signalé.

Les poules recevant un régime séparé (ASL ou ASR) ont eu un indice de consommation ( $g$ aliment/g œuf $+g$ gain de poids) inférieur à celles consommant un aliment complet $(p<0,001)$. Pour cette période (23-42 S), l'IC était en moyenne de $3,38,2,61,2,37$ respectivement pour l'AC, l'ASL et I'ASR. Le rendement énergétique (Kcal EM/g $œ u f+g$ gain de poids) varie dans le même sens que l'IC d'aliment. II a été de 8,44 pour l'AC, 6,69 pour l'ASL et 6,29 pour l'ASR. Le rendement protéique (g protéines/g œuf $+g$ gain de poids) a été de $0,55,0,57$ et 0,42 respectivement pour l'AC, l'ASL et l'ASR.

\section{DISCUSSION}

Les observations faites au cours de cette étude sur les poulettes en croissance et les poules en phase de production recevant une alimentation séparée montrent que les animaux de souche ponte, en situation de choix, ont un comportement alimentaire plus complexe que les poulets de chair (41).

Chez la poulette en période d'élevage, l'utilisation d'un système d'alimentation offrant un libre choix entre une source énergétique et une source protéique a permis d'obtenir une croissance pondérale identique à celle mesurée chez les poulettes consommant un aliment complet, sauf au cours des 3 semaines ayant suivi l'opération de débecquage effectuée au début de la 10 e semaine d'âge. Cette opération a entraîné une diminution importante de la consommation d'aliment complémentaire granulé qui est passée de $34 \mathrm{~g} /$ poulette/j avant l'épointage du bec (5-9 S) à $10 \mathrm{~g} /$ poulette/j après cette opération (10-8 S). En revanche, le niveau d'ingestion du maïs était maintenu, voire accru. Ainsi, le complémentaire qui représentait 50 à 60 p.100 de l'ingéré total avant le débecquage n'en constituait plus que 17,5 p.100 après l'opération. Cette modification du choix alimentaire a fait diminuer la concentration protéique de la ration de $22-28$ p.100 (5-9 S) à 15-16 p.100 (10-18 S).

Si la surconsommation de protéines observée au cours de la première période correspond assez bien aux résultats déjà signalés par d'autres auteurs $(22,24,37)$, l'effet important du débecquage sur le niveau d'ingestion du granulé protéique reste difficile à expliquer. Plusieurs études ont également montré que le débecquage des poulettes entraîne une réduction de l'ingéré et une croissance pondérale plus faible (11). Cet effet persiste généralement pendant 5 semaines et est plus accentué avec un aliment présenté en granulés (10). La douleur et la gêne induites par la section du bec ont généralement été évoquées pour expliquer cette réduction de l'ingestion. 
TABleau IV

Production, consommation moyenne d'aliments et indices de consommation au cours de la période 23-42 S $(\mathbf{n}=\mathbf{5 0}$ poules/traitement)

\begin{tabular}{|c|c|c|c|c|c|c|}
\hline Production & $A C(a c)$ & $A C$ (as) & ASL (ac) & ASL (as) & ASR (ac) & ASR (as) \\
\hline $\begin{array}{l}\text { Taux de ponte }(\mathrm{p} .100) \\
\text { Poids moyen de l'oeuf }(\mathrm{g}) \\
\text { Masse d'ceufs (g/poule/j) }\end{array}$ & $\begin{array}{c}75,4 a \\
57,8 \pm 4,0 a \\
43,6 a\end{array}$ & $\begin{array}{c}74,2 a \\
58,7 a \pm 3,8 a \\
43,6 a\end{array}$ & $\begin{array}{c}72,0 \mathrm{a} \\
59,4 \mathrm{a} \pm 4,0 \mathrm{a} \\
42,7 \mathrm{a}\end{array}$ & $\begin{array}{c}69,7 a \\
60,1 \pm 3,9 a \\
41,9 a\end{array}$ & $\begin{array}{c}74,7 a \\
57,6 \pm 4,3 a \\
43,0 a\end{array}$ & $\begin{array}{c}68,3 a \\
57,6 \pm 4,5 a \\
39,3 a\end{array}$ \\
\hline \multicolumn{7}{|l|}{ Consommation } \\
\hline $\begin{array}{l}\text { Céréale (g/anim./j) } \\
\text { Complément }(\mathrm{g} / \mathrm{anim} / \mathrm{j}) \\
\text { Coquilles (g/anim. } / \mathrm{j}) \\
\text { Total (g/anim./j) } \\
\text { p. } 100 \text { complément }\end{array}$ & $\frac{7}{155 a}$ & $\frac{-}{154 a}$ & $\begin{array}{l}58,1 b \\
50,3 a \\
8,50 a \\
117 b \\
43 \%\end{array}$ & $\begin{array}{l}63,0 \mathrm{ab} \\
48,3 \mathrm{a} \\
7,95 \mathrm{a} \\
119 \mathrm{~b} \\
40,5 \%\end{array}$ & $\begin{array}{l}62,0 a b \\
29,7 b \\
7,89 a \\
100 c \\
29,8 \%\end{array}$ & $\begin{array}{l}65,9 \mathrm{a} \\
29,1 \mathrm{~b} \\
6,97 \mathrm{a} \\
102 \mathrm{c} \\
28,5 \%\end{array}$ \\
\hline $\begin{array}{l}\text { Protéines (g/arim.j) } \\
\text { Energie (koal/ EM/anim./j) } \\
\text { Calcium (g/anim./j) }\end{array}$ & $\begin{array}{l}25,0 a \\
387 a \\
5,92 a\end{array}$ & $\begin{array}{l}24,9 a \\
385 a \\
5,88 a\end{array}$ & $\begin{array}{l}25,7 \mathrm{a} \\
292 \mathrm{~b} \\
4,79 \mathrm{~b}\end{array}$ & $\begin{array}{l}25,4 a \\
304 b \\
4,52 b c\end{array}$ & $\begin{array}{l}17,8 \mathrm{~b} \\
263 \mathrm{~b} \\
3,93 \mathrm{~cd}\end{array}$ & $\begin{array}{l}17,9 b \\
274 b \\
3,56 d\end{array}$ \\
\hline \multicolumn{7}{|l|}{ Indice de consommation } \\
\hline $\begin{array}{l}\text { IC aliment } \\
\text { (g aliment } / g \text { œuf }+g \text { gain) }\end{array}$ & $3,40 a$ & $3,37 a$ & $2,58 \mathrm{~b}$ & $2,64 \mathrm{~b}$ & $2,24 \mathrm{~b}$ & $2,49 b$ \\
\hline $\begin{array}{l}\text { Rendement énergétique } \\
\text { (kcal EM/g œuf }+g \text { gain) }\end{array}$ & $8,47 a$ & $8,40 \mathrm{a}$ & $6,47 \mathrm{~b}$ & $6,93 \mathrm{~b}$ & $5,91 \mathrm{~b}$ & $6,72 b$ \\
\hline $\begin{array}{l}\text { Rendement protéique } \\
\text { (g protéines/g ceuf }+g \text { gain) }\end{array}$ & $0,55 a$ & $0,54 a$ & $0,57 \mathrm{a}$ & $0,57 a$ & $0,40 \mathrm{~b}$ & $0,44 b$ \\
\hline
\end{tabular}

Les moyennes figurant sur la même ligne et portant la même lettre ne sont pas significativement différentes $(p>0,05)$.

Tableau $\mathbf{V}$

Ingestion moyenne d'eau et rapport eau/aliment ( $\mathbf{n}=\mathbf{5 0}$ poules/traitement)

"It:men

Consommation d'eau (g/anim./i)

$24 \mathrm{~S}$

$32 \mathrm{~s}$

$40 \mathrm{~S}$

Ratio eau/aliment

$24 \mathrm{~S}$
$32 \mathrm{~S}$

$40 \mathrm{~S}$
AC (as) ASL (ac)

ASL (as)

ASR (ac)

ASR (as)

$A C$ (ac)

$225 a$

267a

$284 a$

$361 \mathrm{a}$

$356 a$

$276 a$

$390 \mathrm{a}$

$344 a$

$275 a$
$334 a$

$334 a$

$254 a$

$386 \mathrm{a}$

$336 \mathrm{a}$
$3,00 \mathrm{~b}$

$3,48 a b$

$3,14 b$

$2,68 \mathrm{~b}$

4,03a

$3,14 \mathrm{~b} \quad 3,23 \mathrm{~b}$

$2,82 b \quad 2,74 b$

$3,21 \mathrm{~b}$

Les moyennes figurant sur la même ligne et portant la mêrne lettre ne sont pas significativement différentes $(p>0,05)$.

De plus, les volailles disposent au niveau du bec d'un ensemble de mécanorécepteurs qui jouent un rôle capital dans la prise et le transport des particules alimentaires vers la cavité buccale $(2,15,23)$. Ces récepteurs permettent à l'animal d'opérer une discrimination très fine des particules alimentaires; tout dommage au bec entraîne une importante modification des capacités sensorielles de l'animal (1) et peut être de ses choix alimentaires?

En dehors de la période 10-12 $\mathrm{S}$, la forte réduction du taux d'aliment protéique n'a pas altéré la croissance des poulettes ; cela suggère que le choix alimentaire des animaux pendant cette période était probablement compatible avec leurs besoins de croissance. Le taux de protéines dans la ration de 15-16 p.100 correspond assez bien aux recommandations nutritionnelles de l'INRA (1984) et du sélectionneur (ISA, 1990) pour les poulettes d'élevage.

En période de ponte, la distribution d'un régime séparé, constitué de mails, d'un aliment complémentaire en granulé et d'une source particulaire de calcium, n'a pas eu 
d'effet négatif sur la production des poules. Cependant, on a observé que les poulettes ayant reçu l'AS pendant la période pré-ponte montrent pendant les 8 premières semaines de ponte un niveau de production inférieur à celles élevées avec l'AC. Ainsi, alors que les poules élevées avec l'AC atteignent à $21 \mathrm{~S}$ d'âge un taux de ponte de 25 p.100, celles ayant reçu l'AS ont un niveau de ponte de 5 p.100. Cette différence est plus le reflet d'un retard de la maturité sexuelle qu'un effet per se du type d'alimentation pré-ponte. En effet, on a montré que les perturbations du débecquage ont entraîné chez ces animaux un poids vif à $18 \mathrm{~S}$ plus faible (1 $524 \mathrm{~g}$ ) que ceux ayant reçu l'AC $(1600 \mathrm{~g})$. Ces poules ont rattrapé leur retard pondéral au bout de 10 semaines de ponte. Pendant cette période, elles ont dû mobiliser une partie relativement importante des nutriments ingérés pour leur croissance corporelle, aux dépens de la production d'œufs.

L'ensemble des résultats analysant les paramètres de production ont montré qu'il n'y a pas eu d'interaction significative entre les systèmes d'alimentation (AS ou $\mathrm{AC})$ en "phase poulette" et en "phase ponte" conformément aux résultats de Karunajeewa et Tham (21) et Robinson (32). Cowan et Michie (8) ont également observé que l'AS chez les poulettes n'a pas d'effet négatif sur les performances ultérieures de ponte. Cela suggère que l'AS peut être introduite à l'entrée en ponte sans risque de baisse de performance, même si les poulettes n'ont pas été adaptées à ce mode d'alimentation pendant la phase d'élevage.

Certains résultats obtenus en chambres climatiques signalent qu'en température élevée $\left(25-35^{\circ} \mathrm{C}\right)$, l'AS permet d'obtenir un taux de ponte supérieur à l'AC, au moment de l'entrée en ponte (38). Dans la présente étude, cet avantage de l'AS n'a pas été observé. Cela pourrait s'expliquer par l'évolution favorable de la température qui a été, en moyenne, de $27-28^{\circ} \mathrm{C}$ et dont les maxima ont rarement atteint $34-35^{\circ} \mathrm{C}$ (fig. 1). Des essais en climat plus chaud et plus humide (Abidjan, Douala) ou en condition sèche et très chaude (Sahel) pourraient permettre de vérifier cet avantage.

Au cours de cette expérience, les poules ayant eu libre accès au maïs et à l'aliment protéique ont montré une surconsommation de protéines entraînant une augmentation du poids moyen des ceufs mais sans augmentation du taux de ponte ou de la masse d'œufs produite. Beaucoup de doute demeure donc quant à la capacité de la poule pondeuse à "optimiser" son choix alimentaire en fonction de ses besoins de production $(16,22)$ contrairement aux poulets de chair qui ont montré une assez bonne aptitude à réguler leur ingéré nutritionnel en situation de choix (41). Les performances obtenues avec le régime rationné (ASR) montrent que cette surconsommation correspond à un gaspillage et qu'elle peut être corrigée par la distribution d'une quantité limitée d'aliment complémentaire. Toutefois, le rationnement du complémentaire à $30 \mathrm{~g} / \mathrm{anim}$./j semble quelque peu sévère vu le poids moyen des ceufs légèrement plus faible que ceux obtenus avec l'aliment complet. Dans la pratique, on pourrait proposer la distribution à volonté du complémentaire de type $\mathrm{C} 1$ pendant les 8 premières semaines de ponte (adaptation), puis le rationnement à $35 \mathrm{~g}$ par poule et par jour pendant le reste de la période de ponte. Le niveau de consommation observé sur AC semble très élevé (155g) et pourrait être dû à un gaspillage d'aliment.

Les poules rationnées en aliment complémentaire à 30 $\mathrm{g} / \mathrm{j}$ ne reportent pas leur appétit sur le maïs, comme on pouvait le supposer ; la consommation de la plupart des nutriments en ASR est donc globalement plus faible qu'en ASL. La réduction de l'ingéré énergétique en $A S$ (ASR et à moindre titre ASL) comparativement à l'AC, sans réduction significative des performances, conduit à une amélioration de l'efficacité d'utilisation de l'énergie: (pour l'ASR et l'ASL) et des protéines (pour l'ASR). L'amélioration de l'indice de consommation de la ration en AS a déjà été signalée par Kiiskinen (22) et Karunajeewa (20). Chah et Moran (6) expliquent ce phénomène par un meilleur ajustement chronologique entre l'ingestion de nutriments et le cycle de formation de l'œuf.

Du fait de son importance économique, l'évolution des caractéristiques de la coquille avec les régimes alimentaires a fait l'objet de nombreuses études. En effet, Smith (40) indique que la fréquence de casse pendant le transport est très faible pour les cufs ayant une coquille de $0,35 \mathrm{~mm}$ d'épaisseur mais quasi totale pour les coquilles mesurant seulement $0,25 \mathrm{~mm}$ d'épaisseur. La plupart des travaux menés sur l'alimentation calcique séparée ont montré une amélioration du poids, de l'épaisseur, et donc une meilleure résistance à la casse des coquilles $(4,27$, $29,30)$ du fait d'une ingestion plus élevée de calcium ou d'une meilleure utilisation du calcium ingéré. Chez les poules disposant d'un choix alimentaire plus large (énergie, protéines, calcium), plusieurs auteurs n'ont pu observer cette amélioration $(6,22,24)$. Au cours de la présente étude, bien qu'aucun avantage apparent n'ait été observé au niveau des coquilles de l'ASL et de l'ASR, le ratio quantité de coquille déposée/ingéré calcique permet de déduire une meilleure utilisation du calcium dans les régimes séparés.

\section{CONCLUSION}

Les résultats de cette étude montrent qu'il est possible, en milieu tropical, d'utiliser un régime fractionné pour alimenter les poules en ponte sans incidence négative sur leur niveau de production. Il apparait toutefois nécessaire de rationner la source de protéines en vue d'éviter un gaspillage. Un apprentissage préalable en période d'élevage ne semble pas indispensable.

En période pré-ponte, l'utilisation du système d'AS permet une croissance des poulettes comparable à l'AC. Cependant, le débecquage qui induit une perturbation des choix alimentaires a, dans les conditions de l'étude, réduit la vitesse de croissance et entraîné un retard 
d'entrée en ponte des poulettes recevant une alimentation séparée sans toutefois affecter la production ultérieure en ponte. La tendance des poulettes à surconsommer les protéines, l'incidence encore inexpliquẻe du débecquage et la difficulté de rationner un animal en pleine croissance, imposent que des informations supplémentaires soient acquises sur l'utilisation de l'AS pendant la phase pré-ponte.

En définitive, l'utilisation de la technique d'AS pourrait apparaître comme une solution économique pour nourrir les poules en ponte en milieu tropical. En effet, la perspective de valorisation directe des surplus de céréales disponibles sur l'exploitation devrait aboutir à la réduction d'achats d'aliments composés et à la diminution des coûts de production.

\section{REMERCIEMENTS}

Cette étude a bénéficié d'un financement du Fonds d'Aide et de Coopération (FAC) dans le cadre du programme "Recherche-développement pour l'association agriculture-élevage en zone de savane". Les compléments protéiques utilisés ont été mis à notre disposition par l'UFAC (Vigny, France).

\section{BIBLIOGRAPHIE}

1. APPLEBY M.C., HUGHES B.O., ELSON H.A., 1992. Poultry production systems: behaviour, management and welfare. Wallingford, U.K. $\mathrm{CAB}$ International, $238 \mathrm{p}$.

2. BERKOUDT H., 1980. The morphology and distribution of cutaneous mechanoreceptors in bill and tongue of mallard (Annas platyrynchos, L.). Neth. J. Zool., 30: 1.34.

3. BONNET Y., MONGIN P., 1965. Mesure de la surface de l'œuf. Annls Zootech., 14 (4): 311-317.

4. BRISTER R.D., LINTON S.S., CREGER C.R., 1981. Effect of dietary calcium sources and particle size on laying hen performance. Poult. Sci., 60: 2648-2654.

5. CARRE B., ROZO E., 1990. La prédiction de la valeur énergétique des matières premières destinées à l'aviculture. Prod. anim., 3 (3) : 163-169.

6. CHAH C.C., MORAN Jr, E.T., 1985. Egg characteristics of high performance hens at the end of lay when given cafetaria access to energy, protein and calcium. Poult. Sci., 64: 1696-1712

7. CLASSEN H.L., SCOTT T.A., 1982. Self-selection of calcium during the rearing and early laying periods of white leghorn pullets. Poult. Sci., 61: 2065-2074.

8. COWAN P.J., MICHIE W., 1979. Choice feeding of the fowl during rearing and lay. Wld Rev. Anim. Prod., 15 (3): 47 50.

9. COWAN P.J., MICHIE W., ROELLE D.J., 1978. Choice feeding of the egg-type pullet. Br. Poult. Sci., 19: 153-157.

10. DEATON J.W., LOTT B.D., MAY J.D., 1988. Effect of beak trimming on body weight and feed intake of broiler roasters fed pellets or mash. Poult. Sci. 67: 1514-1517.
11. DUNCAN I.J.H., GILLIAN S.L., ELAINE SEAWRIGHT, BREWARD J.,1989. Behavioural consequences of partial beak amputation (beak trimming) in poultry. Br. Poult. Sci., 30: 479-488.

12. EMERY D.A., VOHRA P., ERNST R.A., BURGER R.E., 1990. Egg production and shell quality as influenced by choice-feeding of hens. Appl. Agric. Res., 5(1): 70-74.

13. EMMERSON D.E., DENBOW D.M., HULET R.M., 1990. Protein and energy self-selection by turkey hens: reproductive performance. Br. Poult. Sci., 31(2): 283-292.

14. FARREL D.J., HAMID R.J., HUTAGALUNG R.I., 1981. Free choice feeding of laying hens in the humid tropics. Trop. Anim. Prod., 6 (1): 22-29.

15. GENTLE M.J., 1979. Sensory control of food intake. In: Boorman K.N., Freeman B.M. eds, Food intake regulation in poultry. Edinburgh, Scotland, U.K., Br. Poult. Sci. Ldt, p. 259-273.

16. HUGHES B.O., 1984. The principles underlying choice feeding behaviour in fowls - with special reference to production experiments. Whd's Poult. Sci.J., 40 (2): 141-150.

17. Institut national de recherches agronomiques, 1984. L'alimentation des monogastriques : porc, lapin, volailles. Paris, France, INRA, 282 p.

18. Institut de sélection animale, 1990. Guide d'élevage des pondeuses Isabrown. Lyon, France, ISA, 40 p.

19. JANSSEN W.M.M.A., 1988. European table of energy values for poultry feedstuffs. Wageningen, Pays-Bas, Grafischbedrijf Ponson et Looijen bv Wageningen, 2nd ed., p. 1-13

20. KARUNAJEEWA H., 1978. The performance of cross-bred hens given free choice feeding of whole grains and a concentrate mixture and the influence of source of xantophylle on yolk colour. Br. Poult. Scit, 19: 699-708.

21. KARUNAJEEWA H., THAM S.H., 1984. Choice feeding of the replacement pullet on wholc grains and subsequent performance on laying diets. Br. Poult. Sci., 25 (1): 99-109.

22. KIISKINEN T, , 1987. Influence of choice feeding on the performance of growing pullets and laying hens. Annls Agric, Fenniae, 26 (2): 131-144:

23. KUENZEL W.J., 1989. Neuroanatomical substrates involved in the control of food intake. Poult. Sci., 68: 926-937.

24. LENNARD R.M., ROLAND D.D.A., 1981. The influence of time of dietary calcium intake on shell quality. Poult. Sci., 60: 2106-2113.

25. LEESON S., SUMMERS J.D., 1977. Dietary self-selection by poultry. Feedstuffs, 49 (19): 21-24.

26. LEESON S., SUMMERS J.D., 1979. Dietary self-selection by layers. Poult. Sci., 58: 646-651.

27. MARCH B.E., AMIN M., 1981. Dietary limestone versus extra-dietary oyster shell as calcium supplements to different layer diets. Poult. Sci., 60: $591-597$.

28. NIR I.. MELCION J.P.. PICARD M., 1990. Effect of particle size of sorghum grains on feed intake and performance of young broilers. Poult. Sci. 69: 2177-2184.

29. NYS Y., 1990. Qualité de la coquille de l'œuf. Revue Alim. anim., 440: $43-44$.

30. PICARD M., 1985. Heat effects on the laying hen: Protein nutrition and food intake. Proc. 5th Europ. Symp. Poult. Nut. Maale Hachamisha, Istael, 27-31 October 1.985 , p. 65-72.

31. PICARD M., ANTOINE H., SAUVEUR B., 1986. Influence de l'alimentation calcique séparée sur l'ingéré énergétique de la poule pondeuse soumise à un stress thermique. $I n$ : Larbier M. ed., Proc. of the 7th Europ. Poult. Conf., Paris 24-28 août 1986. Paris, WPSA, Branche Française, p. 406-410. 


\section{T. Yo M. Picard H. Guerin P. Dauvilliers}

32. ROBINSON D., 1985. Performance of laying hens as affected by split time and split composition dietary regimens using ground and unground cereals. Br. Poult. Sci, 26 (3): 299-309.

33. SAUVEUR B., 1988. Reproduction des volailles et production d'œufs. Paris, France, INRA, 449 p.

34. SAUVEUR B., 1992. Adaptation des apports alimentaires aux variations journalières des besoins en calcium et phosphore de la poule. Prod. anim., $5(1): 19-28$

35. SAUVEUR B., MONGIN P., 1974. Effects of time-limited calcium meal upon food and calcium ingestion and egg quality. Br. Poult. Sci., 15: 305-313.

36. SCOTT T.A., BALNAVE D., 1985. Choice feeding of pullets at high environmental temperature. In: Cumming R.B. ed, Recent advances in animal nutrition in Australia. Proc. Symp. University of New England, November 24-27, 1985, unnumbered part.

YO (T.), PICARD (M.), GUERIN (H.), DAUVILLIERS (P.). Free choice feeding (whole-grain maize + complementary pellet concentrate + oyster shells) of pullets and laying hens in a hot climate. Revue Élev. Méd. vét. Pays trop., 1995, 48 (1): $67-76$

Between the age of 5 and 42 weeks, 300 pullets Isabrown were fed either a control complete mash feed (CMF) or a free choice (FC) between whole grain-maize, oyster shells and a complementary pelleted concentrate offered ad libitum (alFC) or restricted $(30 \mathrm{~g} / \mathrm{hen} / \mathrm{day})$ during the laying period $(r \mathrm{FC})$. Feed intake and growth of pullets were not modified by the diets until debeaking at 10 weeks. Debeaking decreased intake of complementary concentrate from 53 to $17 \%$ of total intake. At 18 weeks, FC pullets weighed less than CMF $(1,524$ and $1,600 \mathrm{~g}$, respectively) and began to lay a little later: laying intensity at 21 weeks was 5 and $25 \%$ respectively. Between 23 and 42 weeks of age, laying production and egg quality were not significatively influenced by the diet. Total feed intake of FC hens was significatively lower than for the CMF control group. Thus, the efficiency of energy and protein utilisation was better with $\mathrm{FC}$. al $\mathrm{FC}$ tended to overconsume the complementary feed $(50 \mathrm{~g} / \mathrm{day} / \mathrm{hen}$ instead of 30 g/day with $r \mathrm{FC}$ ) without improving production. Under these conditions, free choice feeding with a restriction on the complementary feed promotes the direct use of locally-produced cereals, saving on transportation, grinding, mixing and pelleting costs of feeds for laying hens.

Key words: Laying chicken - Feeding system - Food resource - Growth Oviposition - Egg character - Tropical climate - Côte d'Ivoire.
37. SCOTT T.A., BALNAVE D., 1986. The influence of dietary self-selection on performance of young laying pullets under hot and cold environmental temperatures. In: Larbier M. ed, Proc. of the 7th Europ. Poult. Conf., Paris, 24-28 Août 1986, WPSA, Branche Française, p. 401-405.

38. SCOTT T., BALNAVE D., 1988. Comparison between concentrated complete diets and self-selection for feeding sexually-maturing pullets at hot temperatures. Br. Poult. Sci., 29: 613-625.

39. SCOTT T., BALNAVE D., 1989. Response of sexually maturing pullets to self-selection feeding under different temperatures and lighting regimes. Br. Poult. Sci., 30: 135-150.

40. SMITH A.J., 1990. Poultry. London, U.K., The Mcmillan Press Ltd, 218 p.

41. YO T. PICARD M., GUERIN H., DAUVILLIERS P., 1994. Alimentation séparée (céréales graines entières + aliment complémentaire granulé) chez les poulets de chair en climat chaud. Revue Élev. Méd. vét. Pays trop. $47(3): 319-327$.

YO (T.), PICARD (M.), GUERIN (H.), DAUVILLIERS (P.). Alimentación libre (granos enteros de cereales + suplemento granulado + concha de ostra) para pollitas y ponedoras en clima cálido. Revue Élev. Méd. vét. Pays trop., 1995, 48 (1) : 67-76

Trescientas pollas Isabrown recibieron de 5 a 42 semanas una alimentación control completa en harina $(\mathrm{AC})$, 0 una alimentación libre (AS) de granos enteros de maiz, concha de ostra y un suplemento granulado, ad libitum (ASL) o controlado durante la postura (ASR: $30 \mathrm{~g} /$ ponedora/día). Hasta el momento del recorte de pico, a las 10 semanas, la ingestión total de alimento y el crecimiento de las pollitas no fueron modificados por el régimen. Este recorte redujo la proporción de suplemento ingerido por las pollitas en ASL de 53 a 17 p. 100 de la ingestión total. Las pollas en ASL presentaron un peso vivo medio a 18 semanas inferior al de las pollas $\mathrm{AC}\left(\begin{array}{l}1524 \\ \text { y } 1600\end{array}\right.$ pectivamente), con una postura un poco tardía (intensidad de postura a 21 semanas : 5 y 25 p. 100 respectivamente). Durante el período de 23 - 42 semanas, los rendimientos de postura y las características de los huevos no fueron influenciados en forma significativa por el régimen alimenticio. Las cantidades globales de alimentos consumidos por las gallinas en AS fueron significativamente inferiores a las de las gallinas testigo AC. Por lo tanto, los rendimientos energéticos y proteicos de los regímenes fueron mejorados mediante la alimentación libre. Las gallinas ASL presentaron una tendencia a un sobre consumo del suplemento alimenticio ( $50 \mathrm{~g} / \mathrm{d}$ ía/gallina en vez de $30 \mathrm{~g} /$ día por gallina ASR), sin mejora alguna en las producciones. En esas circunstancias, la alimentación libre controlada permite una utilización directa y eficaz de los cereales producidos localmente, mediante una reducción de los gastos de transporte, trituración, mezcla y granulación de los alimentos de las ponedoras.

Palabras clave : Gallina ponedora - Sistema de alimentación - Recurso alimentario - Crecimiento - Oviposición - Caracteristica del huevo Clima tropical - Côte d'Ivoire. 